\title{
HEAT TRANSFER FOR THE FLOW THROUGH A PIPE
}

\author{
BY \\ D. Y. KASTURE
}

Marathwada University, Aurangabad, Maharashtra, India

\begin{abstract}
The heat flux per unit length through the wall of a straight pipe of arbitrary but uniform cross section is shown to be the product of the constant pressure gradient and the volume flux, when a steady Poiseuille flow of a viscous incompressible fluid is maintained through it, and its wall is kept at a constant temperature. Bounds on the heat flux are obtained using the methods of isoperimetric inequalities.
\end{abstract}

1. Introduction. Consider the steady Poiseuille flow of a viscous incompressible fluid through a straight pipe of uniform but arbitrary cross section, when its wall is maintained at a constant temperature $T_{0}$. Taking the $z$-axis along the length of the pipe, neglecting the variations of the coefficient of viscosity $\mu$ and the conductivity $k$ of the fluid with temperature, but taking into account the dissipation of energy due to viscosity, the equations for the velocity $w(x, y)$ along the pipe, and the temperature $T(x, y)$ in it, are [2, p.39]:

$$
\begin{gathered}
\nabla^{2} w=-P / \mu, \\
\nabla^{2} T=-(\mu / k)\left(w_{x}^{2}+w_{y}^{2}\right)
\end{gathered}
$$

in $S$, while

$$
w=0, \quad T=T_{0} \quad \text { on } \partial S .
$$

Here $S$ is the cross sectional region of the pipe bounded by a closed curve $\partial S$ and $-P(P>0)$ is the constant pressure gradient along the pipe.

It is possible (i) to express the mean temperature in $S$, the mean temperature gradient over $\partial S$, the mean Nusselt number, and the heat flux $H$ across the wall per unit length of the pipe in terms of certain integrals of $w$, without requiring a pointwise solution of (1.2), (1.3), (ii) to obtain the bounds on them, and thus (iii) to develop a qualitative theory of heat transfer. To illustrate this, we obtain an expression for the heat flux $H$ in the next section.

2. The heat flux $H$. This is the most important quantity. It is given by

$$
H=\left|\int_{\partial S} k(\partial T / \partial n) d s\right| \text {. }
$$

Received September 26, 1989. 
Using (1.1)-(1.3) and the Green's identities, we obtain

$$
\begin{aligned}
H & =k\left|\int_{S} \nabla^{2} T d s\right|=\mu\left|\int_{S}\left(w_{x}^{2}+w_{y}^{2}\right) d S\right| \\
& =\mu\left|\int_{\partial S} w(\partial w / \partial n) d s-\int_{S} w \nabla^{2} w d S\right| .
\end{aligned}
$$

Therefore

$$
H=P Q
$$

where

$$
Q=\int_{S} w d S
$$

is the volume flux of fluid through $S$. Equation (2.2) is an expression for an obvious energy balance.

3. General results on heat transfer. Since $w$ and hence $Q$ is proportional to $P / \mu$, we may write $(2.2)$ in the form

$$
H=\left(P^{2} / \mu\right) q,
$$

where $q$ is a purely geometric quantity. When $S$ is simply connected, $q$ is $1 / 4$ of the torsional rigidity [1, p. 64]. Thus several standard properties and bounds on torsional rigidity (which is proportional to $q$ ), for example, $[1, \mathrm{pp} .64,67,150$, 152], are directly applicable to (3.1) when $P^{2} / \mu$ is fixed. Some of the results thus obtained when $P, \mu$ are fixed, are given below.

(i) $H$ is independent of $k$, the conductivity.

(ii) For a given area of cross section, the circular pipe offers the maximum $H$.

(iii) $H$ is an increasing functional of of the domain $S$ (i.e., $S_{1} \subseteq S_{2}=>H_{1} \leq$ $H_{2}$ ) .

(iv)

$$
H \geq\left(P^{2} S^{2} / 8 \pi \mu\right)\left[1-2 \beta^{2}\left(1-\beta^{2}\right)^{-1}-4 \beta^{4}\left(1-\beta^{2}\right)^{-2} \log \beta\right],
$$

where $S$ is the area of $S, L$ is the length of $\partial S$, and $\beta=1-4 \pi S / L^{2}$. Inequality (3.2) becomes an equality for a circle with $\beta=0$.

(v)

$$
H>P^{2} S^{3} / 3 L^{3} \mu \text {. }
$$

Statements (ii) and (iii) hold for the torsional rigidity, and therefore, in view of (3.1), they hold for $H$. (iv) and (v) are direct consequences of (3.1) and the corresponding results for the torsional rigidity due to Payne-Weinberger and Polya [1, p. 150]. These results give some lower bounds on $H$ in terms of the geometric constants of the domain, and the physical constants $P, \mu$.

3.1. Trap-domains. Since $Q$ (and hence $q$ ) is explicitly known for several standard domains such as the regions bounded by (i) a circle, (ii) a pair of concentric or eccentric circles, (iii) an ellipse, (iv) an equilateral triangle, and (v) a rectangle [4], a semicircle, and its diameter [3], $H$ is known explicitly for these domains from (2.2) or (3.1) without requiring a pointwise solution of (1.2), (1.3). An arbitrary region $S$ may be trapped between any two such best fitting standard domains $S_{1}, S_{2}$ in the 
sense that the difference of the areas $\left|S-S_{i}\right|, i=1,2$, is as small as possible and $S_{1} \subseteq S \subseteq S_{2}$. Then using the result (iii) above upper and lower bounds for $H$ are easily obtained. For example, when $S_{i}$ are circles of radii $r_{i}, i=1,2$, so that $2 r_{1}$ and $2 r_{2}$ indicate 'the width' and the 'length' of $S$ respectively,

$$
\left(\pi P^{2} / 8 \mu\right) r_{1}^{4} \leq H \leq\left(\pi P^{2} / 8 \mu\right) r_{2}^{4} \text {. }
$$

Obviously the upper bound in (3.4) is never better than the isoperimetric bound given by result (ii) above.

Acknowledgment. The author would like to thank Professor H. F. Weinberger and the referee for their useful comments on the earlier drafts of this paper; and to the National Centre for Science information, Bangalore, India, for regularly supplying computerised information about the current research in this area.

\section{REFERENCES}

[1] C. Bandle, Isoperimetric Inequalities and Applications, Pitman, New York, 1980

[2] J. L. Bansal, Viscous Fluid Dynamics, Oxford and I. B. H. Co., Calcutta, India, 1977

[3] E. M. Sparrow and A. Haji Sheikh, Flow and heat transfer in ducts of arbitrary shape with arbitrary thermal boundary conditions, Trans. ASME Ser. C. J. Heat Transfer 88 (4), 351-358 (1966)

[4] F. White, Viscous Fluid Flow, McGraw-Hill, New York, 1974, p. 118 